\title{
Exploration on Current Art Design Elements in Digital Media Era
}

\author{
Hu Jijun \\ Shanghai Institute of Visual Art, Shanghai201620, China
}

\begin{abstract}
In the digital information era with fierce competition, rapid, accurate and efficient transference of information has been the key factor for enterprises to win in the market. Analyzing from the medium and ways of information delivery, no matter two-dimensional figures,text composition, mass production under print medium or the popular internet contact and communication needs digital technique. And applying digital delivery has tended to be the main stream. Depending on the contemporary art of digital media to generate abstract and virtual graphical symbol language has the characteristic of infinite scalability and innovation.
\end{abstract}

Key words: digital technique, media, art design

\section{INTRODUCTION}

Gestalt psychology proposes that the people have higher aesthetic demand after the basic requirements are met. And the aesthetic demand needs to have direct and close correlation with vision, audition, smell, gustation and touch of people. The relationship between the people and visual product design not only is the simple application, but also has emotional communication of people and people, and people and things.

Different ages and different backgrounds have different aesthetic features and artistic characteristics, which means that there are different design aesthetic ideas and experience.
Under the situation that information develops rapidly, various competitions and pressure is more and more fierce, which brings massive and new aesthetic requirements for people. The most typical feature visual figure in interactive digital era is exercise. Dynamical graphics can enhance the visual effect and tension of graphics. In interactive information era, digital interactive graphic design and interactive graphic design is the necessary development tendency. Objectively, digitization is the development stage of promoting aesthetic appreciation to higher performance level. Subjectively, it is a purity pursuing harmonious beaut, which means to pursue clear, plain and intense visual impact, and huge and invisible tension. And it combines the formal movement rule of beauty with the modern digital interactive graphics.

The thrive of art design caused by a new times media depends on the establishment and construction of the current art aesthetic experience category. As comprehensive and diversified art design, the information delivery of digital times graphical language has great artistic fascination, which has the following manifestations.

The unique synaesthetic feeling form of digital graphic language provides a new visual feast and aesthetic experience for the people with great pressure. There are scientific researches indicating that for the sensory organs of normal people, $95 \%$ of the received information depends on vision and auditory organ. Therefore, in five senses of people, vision and audition has definite advantages and 
extraordinary significance. However, in order to remedy the deficiency of sensory irritation, the designers need to enhance the irritation of other sensory organs. For example, it is very important to add the factors of audition, touch, smell and taste to vision design for the blinders.

(1) Digital graphic language is a new mode of thinking which expands and enriches cognitive style and aesthetic demand of the people. In the high-tech era with intensive information, the specific mode of thinking of the people is a digital graphic thinking mode based on all shapes and colors. It not only is different from the single-strand thinking mode in text printing era, but also is different from concrete thinking in daily life. Digital graphic language has great charm, the reason for which is that it uses shape and color as main thinking objects and the important thinking mode to achieve a common visual language, and finally uses it as the thinking results. Therefore, the thinking manifestation mode of shape and color runs through the thinking activities of the people.

For aesthetic ideal, digital graphic language pursues the novel and various visual and auditory sensory stimulation and enjoyment. Compared with visual impact, auditory and psychological impact and motivational power of touch, aesthetic experience of virtual digital graphic language not only has the above aesthetic experience, but also has the following new features from the characteristics of digital graphics and manifestation mode of digital graphic language.

(2) Timeliness of aesthetic experience

The unique art and design aesthetic atmosphere generated by the application of digital virtual technology and continuous development of new functions of computers not only breaks through the strict boundary of people and people, and people and objects, but also realizes real-time and instant communication. Under the situation, the visible world is not real any longer, and the invisible world is not the dream any longer, which directly makes that the distance of art design and life reduce. For example, the online shopping makes many impossible things become the fact. The information of manufactures and consumers can be achieved by reading the intermediary website, which can meet the requirements in different places. And the people can achieve the required things at any time. In addition, when there are new news, events and designs, someone publishes it on the website for the first time, and we can achieve the latest information by network screen. The most typical example is iPhone. Before iPhone5 hit the market, many industries had various designs, which is the great charm of network information. The people can always trace, survey and research their favorite products, which makes the world more and more transparent.

(3) Interaction of art aesthetic experience

Martin Heidegger1, a German philosopher, said that when we understand something, we use the consciousness of the activity to participate actively and think creatively rather than using blank brain to accept passively. Therefore, the interaction of aesthetic experience requires the people to participate and think actively.

Digital technique not only provides good artistic practice technical condition for reception aesthetics and aesthetics, but also provides new visual enjoyment media for the audience. The audience can make thinking graphic organization on the objects according to there individual aesthetic feelings, rich aesthetic experience and extraordinary aesthetic comprehension. Then, they modify the graphic symbol language and recreate to make them 
create aesthetic concept meeting their aesthetic interest under the background of digital life, and create new and individual graphic symbol language fitting their aesthetic ideal, which achieves the aesthetic realm of pleasing ears and eyes, pleasing mind and emotion , and pleasing will and spirit.

Therefore, in some sense, digital graphic design shows the aesthetic ideal of decentralization of post-structuralism. The boundary between the designers and the audience of digital graphic design works weakens gradually.

(4) Immersion of aesthetic experience Digital technology and digital graphic design can create a special and creative aesthetic space experience. The audience is not a simple information receiver and appreciator under the background of the present digital life, but is a participator recreating and redesigning digital art design. And the audience can achieve a free aesthetic synaesthesia. In virtual digital graphic language design space, the people can completely break away from the influence of some concrete thinking in real life and the constraint and limitation of concrete graphic language, which makes the thinking and vision of people more rich and colorful.

The graphic design inspiration and freedom achieved by aesthetic thinking imagination is not only more individualized, but also is wider. But the boundless thinking imagination only brings an abstract interaction and vitality for the audience. But the graphic design in digital virtual space brings a concrete and diversified perception for the audience, which touches various senses of the people, which can achieve easy and efficient interaction of people and people, people and the society, and people and objects.

\section{Art Design under Cultural Diversity}

The society consists of diversified individuality and personalized people, and culture consists of thinking activities of personalized people. If it breaks away from concrete individuals or culture, there is no an abstract people, but there is abstract culture. The diversified situation of design culture has formed with the development of digital era. The diversity of culture provides rich resources for the update and innovation of the current digital society culture.

Diversified culture phenomenon not only can make the life and thinking activities of people more rich and colorful, but also can meet the personal requirements of different individuality. Containing and absorbing different cultures is an important manifestation that the society develops and is full of vitality. The globalization and internationalization of economy makes the communication of different nationalities and cultural languages in different countries more and more frequent. They need to refer to the culture achievements of other nations to enrich themselves, which can realize the real diversity.

\section{People-oriented Green Design}

It is an urgent subject for human society to create green design. While design stimulates the consumption, how to keep sustainable development of society is an important problem for the current designers.

Under the condition that green design is extended into digital graphic language, no matter creativity or manifestation method and pattern brings new connotation for the delivery of graphic language. While passing messages, graphic language makes lots of visual disasters 
such as poisonous materials, blinding color, great light and stiff graphics. The human are puzzled by decent visual manifestation patterns and unorganized symbols, and the mind and body is deeply hurt. Therefore, the people enhance applying green design to vision, which inhibits visual pollution and makes graphic language information meet visual order and visual laws. The coordination relationship of graphic symbol and the human is improved, and the delivery pattern meeting green design concept is explored under the view of green design.

Network advertisement meets the ideological feature because of the characteristics of high efficiency, low pollution and energy conservation, and it can be called green design of information delivery of digital graphic language. And it is a new tendency of information delivery of advertisements in the future. High technology is transformed into high emotion, and transforming nature is transferred into adapting to nature, which is the theme of information delivery of the digital graphic language in the future. Green design cannot be separated from energy conservation, environmental protection and ecology. The designers generally apply different expressions to advocate environmental protection and low carbon.

\section{Conclusions}

The development direction of modern art not only is a topic about the future, but also is a topic about the past. It is based on rethinking and summarizing the experience in the past. Only modesty is not enough for art design. Therefore, whether considering from the international perspective or starting from itself, it is the way to adjust the concept and combine the advantages to transform the role from following blindly to guiding.

\section{Reference:}

[1] Shi Jing, Exploration on digital media design and animation characteristics $[\mathrm{J}]$, Art Science and Technology, 2014,07:47+54.

[2] Yi Zhaoyong, Art design under digital media environment [J], Popular Literature, 2014,22:89.

[3] Liu Wei, Analysis on art design under digital media $[\mathrm{J}]$, Scientific and Technical Information ( scientific research), 2007,20:333+410.

[4] Wang Jingbo, Analysis on digital media art education of higher art institutes [J], Art Panorama, 2007,06:143.

[5] Tu Zhongfang, Application of VR technique based on image core to digital media technique design $[\mathrm{J}]$, Journal of Jilin Art College, 2007,04:44-45+54. 